\section{MMPs and rheumatoid synovial fibroblasts: Siamese twins in joint destruction?}

\section{U Müller-Ladner, S Gay}

\section{MMPs and RA-SF act together in matrix degradation; targeting one will provide details about the other}

$\mathrm{n}$ this issue of the Annals, Tolboom and coworkers present functional data on the intensive invasive potential of rheumatoid synovial fibroblasts (RA-SF) into matrix as well as a direct correlation of this destructive property with distinct members of the large matrix metalloproteinase (MMP) family. ${ }^{1}$

Key findings of this study include (a) demonstration of the higher and disease-specific ability of RA-SF-in comparison with other synovial fibroblasts-to grow through artificial matrix; (b) demonstration of a lack of correlation between invasive growth and the rate of fibroblast proliferation; $(c)$ the dominant role of MMP-1, MMP-3, and MMP-10 in this model of a joint destructive process; and $(d)$ the finding of an association between members of the MMP family-namely, MMP-1 and MMP-9, with the diagnosis of rheumatoid arthritis (RA). Basic as well as clinical rheumatologists may feel that the results of the study could have a direct impact on future developments in diagnosis and treatment of RA but also feel the need to discuss a number of questions arising from these interesting data.

\section{EVALUATION OF HUMAN RA JOINT DESTRUCTION: HOW CLOSE ARE WE TO "THE" EXPERIMENTAL APPROACH?}

Unfortunately, nature provides only the human being as a potential "model" for a number of rheumatic diseases, especially for RA. Therefore, the various animal models used in basic science, including antigen-induced as well as mutation-, knockin-, and knockout-induced arthritides, always have the disadvantage of a certain experimental and clinical distance from human disease. On the other hand, all "humanoid" experimental settings have failed to resemble a whole joint completely, leaving each of these approaches with its individual shortcomings. In the present approach, which examines the invasiveness of RA-SF into an artificial matrix (Matrigel) consisting predominantly of collagen type IV, the authors deal specifically with this problem. They note and balance the advantage of the stability and validity of the system against the preselection of MMPs-2, -3, -7, -9, and -10 , which preferentially degrade collagen type IV and not "cartilage collagen" type II. This problem was underlined by the fact that contact with a certain type of matrix can induce differential MMP synthesis. ${ }^{2}$ On the other hand, the present paper together with data derived from similar approaches examining the interaction between RA-SF and articular matrix or cartilage, support, at least in part, the hypothesis that these nonanimal models for RA may be sufficient or preferable for examining the pathophysiology at the sites of invasion and for the comparison with other arthritic synovial fibroblasts.

\section{"Targeting one twin will also provide details about the other"}

For example, Ermis et al evaluated the invasiveness of multicellular spheroids derived from rheumatoid synovial tissue when co-incubated with human articular cartilage $e^{3}$ and obtained similar results to those seen in the SCID mouse model for RA, ${ }^{4}$ showing that destruction of cartilage by matrix MMPs and cathepsins was predominantly driven by RA-SF and not by osteoarthritis fibroblasts. In addition, by demonstrating a lack of correlation between invasion and the rate of proliferation of the RA-SF, the authors contribute significantly to the longstanding discussion of an enhanced rate of proliferation versus a dysbalance of pro- and anti-apoptotic pathways in these cells. ${ }^{56}$

Most notably, the present study ${ }^{1}$ illustrates also the problems that every researcher nowadays encounters in the developing world of high sensitivity and high throughput molecular biology. Thus eters in parallel-that is, more than 10 (of more than 20 known) MMPs that are the measurement of a variety of param- potentially inherent or correlated with the disease and with each other, requires an experienced statistician to clarify which of the different multivariant analyses, such as principal component analyses, analysis of variance, or Bonferroni, needs to be applied. After the first detailed multiparameter studies using differential subtraction techniques to identify genes that characterise the phenotype of $\mathrm{RA}^{-\mathrm{SF}^{7}}$ the introduction of cDNA arrays has further complicated this issue, resulting in the need for adequate normalisation strategies and for mandatory confirmation of the specific differences on different expression levels. ${ }^{8}$ This development resulted also for the first time in defined, method dependent, manuscript submission criteria in a scientific rheumatology journal. ${ }^{9}$

Therefore, a possible answer to the first question might be that we appear to get closer to real life and its complexity but actually move further away from a single experimental setting for $R A$.

\section{MMPS AND RA-SF: HOW "SIAMESE" ARE THEY?}

Among other proteinases such as serine, cysteine, and aspartate proteinases, matrix MMPs have been shown to be essential for degradation of articular matrix as they not only cleave extracellular matrix components ${ }^{10}$ but also cleave each other, resulting in a domino effect of MMP propeptide and MMP activation. ${ }^{10}$ This is further supported by recently discovered extracellular MMP inducers that are closely associated with MMP-1 and MMP-3 synthesis in RA-SF. ${ }^{11}$ In total, about 20 members of the growing family of matrix MMPs are currently known (MMPs 1-3, 7-17, 19-21, 23, and 24), of which some belong to membrane-type (MT) MMPs-for example, MTl-MMP (MMP-14) and MT3-MMP (MMP-16) and to the MMP subfamily of aggrecanases. ${ }^{2}$

Numerous researchers have provided a large body of data showing that MMP-1 and MMP-3 are most important for joint destruction in RA. ${ }^{10}$ The present study underlines this hypothesis by associating MMP- 1 and MMP-3 expression directly with a functional parameter-that is, the number of cells that is actively passing through a matrix transwell system. ${ }^{1}$ In addition, in the past years synovial fibroblasts have not been only demonstrated as a major source of these enzymes but MMP-1 expression has also been linked to sites of erosions. ${ }^{12}$ Although MMP- 1 seems to be the most prominent matrix MMP, almost all other MMPs have been detected in RA synovium ${ }^{13}$ and, as the present article shows, the association of MMP-3 appears to be restricted to invasiveness-probably because it paves 
the way for transmigration of inflammatory cells through basement membrane containing blood vessels into the synovium instead of solely reflecting cartilage destruction-but not to the diagnosis RA. This finding also favours the hypothesis that RA-SF not only participate in joint destruction but also promote inflammation by extensive tissue remodelling. Most recent experiments have shown a link between the new membrane-type MMP family members MT1-MMP (MMP-14) and MT3-MMP (MMP-16), which can cleave extracellular matrix components as well as activate other MMPs, and RA-SF. In RA synovium, abundant expression of MMP-14 and MMP-16 could be demonstrated, with MMP-16 being expressed by synovial fibroblasts and MMP-14 being synthesised both by synovial fibroblasts and CD68 positive osteoclasts and macrophages. ${ }^{13}$ This led to the concept that matrix degradation driven by RA-SF at sites of synovial cartilage invasion is based on a complex consisting of MMP14, tissue inhibitor of matrix metalloproteinase-2 (TIMP-2), and MMP-2. ${ }^{12} 13$

Supporting the conclusions of the present article, ${ }^{1}$ molecular biology showed also that extracellular and intracellular signalling pathways resulting in MMP synthesis can be attributed to and induced in RA-SF. For example, after stimulation by tumour necrosis factor $\alpha$ $(\mathrm{TNF} \alpha), \mathrm{MMP}-8$ was subsequently up regulated in cultured synovial fibroblasts ${ }^{14}$ and MMP-13 was produced after stimulation of various proinflammatory cytokines, including interleukin 1 (ILl) and TNF $\alpha \cdot{ }^{15}{ }^{16}$ On the other hand, it is most likely that the interaction of RA-SF with other cells is critical for long term destruction as MMP-13 was not only associated with raised inflammation parameters ${ }^{15}$ but could also be found in synovial fibroblasts at sites of synovium-cartilage interaction in close proximity to lymphocytes expressing ILl and TNF $\alpha .{ }^{16}$ Both ILl and TNF $\alpha$ may drive the fibroblasts to produce MMPs also on an intracellular signalling level. For example, within the MMP-3 flanking region a binding site sensitive for $\mathrm{TNF} \alpha$ could be identified, which appears crucial for the ILl mediated up regulation of stromelysin gene transcription. ${ }^{17}$ Moreover, intracellular ILl dependent signalling pathways induced production of MMP-1 that was mediated by NF- $\kappa B$. Further experiments may show that this ILl-induced activation of MMP synthesis in RA-SF was restricted to a distinct section within the MMP-1 promoter. ${ }^{18}$ Last but not least, cartilage destruction in RA is a complex interaction of synovial cells with the cartilage itself. As shown by Neidhart et al, the production of ILl by chondrocytes may further enhance the activity of cartilageinvading rheumatoid synovial fibroblasts. ${ }^{19}$ Because Tolboom et al did not examine chondrocytes in their system, ${ }^{1}$ the cartilage mediated part of joint destruction appears lacking.

In summary, it appears that in matrix degradation, RA-SF and MMPs act as Siamese twins, with a key problem still remaining: what cascade of events created their alliance?

\section{MMPS IN RA: MEDIATORS OR MARKERS?}

An intriguing part of the presented article $^{1}$ is the fact that the authors show a significant association of two parameters - that is, MMP-1 and MMP-9, which can be measured easily with current methods on the mRNA and protein level as well as in rheumatoid cells and tissues, with the diagnosis of RA. As this has been calculated from the basis of a rather large number of patients, it will be most interesting to see whether these parameters will facilitate early diagnosis in mini-arthroscopy samples derived from early unclassified arthritides, especially as matrix degrading enzymes can be detected in RA synovium at a very early stage of the disease.$^{20}$ In contrast, the value of determination of MMP-1 and -9 expression in synovial tissue might be restricted to diagnosis itself rather than to evaluation of disease activity. The rationale for this more cautious statement is the fact that although the number of MMP-1 producing cells reflects the degree of synovial inflammation, ${ }^{21}$ other members of the MMP family-for example, MMP-9, appear to be markers for inflammation rather than being specific for RA. This is supported by the fact that MMP-9 is not restricted to synovial fibroblasts, but is also synthesised by endothelial cells, macrophages, and leucocytes. ${ }^{22}{ }^{23}$ Moreover, matrix degradation in the human joint cannot be separated from the obvious degradation of bone by the aggressively growing rheumatoid synovium, in which the fibroblast dependent mechanisms differ from those mediating cartilage degradation. In the former, the cysteine proteinase cathepsin- $\mathrm{K}$, for example, is not only expressed by synovial fibroblasts ${ }^{24}{ }^{25}$ but is also found in synovial macrophages and osteoclasts, supporting the hypothesis that cooperation of these cells, presumably including membrane-type matrix $\mathrm{MMP}-1,{ }^{26}$ is mandatory for fibroblast dependent bone degradation.

Thus, a possible answer to the question posed above might be that there is little doubt about MMPs being "the mediators" but there is still a long way to go before they become a therapeutic or diagnostic marker.

\section{MMPs AND SYNOVIAL FIBROBLASTS: WHICH OF THE "TWINS" IS THE BETTER THERAPEUTIC TARGET?}

Progressive destruction of the structural integrity of articular components is one of the key features of RA pathophysiology, although recent advances in treatment arrest radiological joint destruction for two years, none of the hitherto used disease modifying antirheumatic drugs, including the biological agents, has yet provided long term, problem-free joint protection. Owing to its key role in joint destruction, MMP inhibition has been an attractive strategy but regardless of initial in vivo data obtained from the application of a collagenase inhibitor in an animal model ${ }^{27}$ and of inhibition of cathepsin synthesis in RA-SF by highly specific ribozymes, ${ }^{28}$ none of these approaches has yet produced sufficient results in clinical trials. This outcome may be predominantly due to the fact that in view of the potentially severe side effects, ${ }^{29}$ elimination of only one enzyme involved in matrix degradation may not be sufficient to inhibit the destructive process. $^{30}$

RA-SF, on the other hand, are even more difficult to target, as neither specific markers for these deleterious cells exist, nor do we have substantial data to unveil potential effects of current treatments on this synovial cell type.

Therefore, the answer to the last question is that targeting one of the twins will probably provide certain details about the other one.

Ann Rheum Dis 2002;61:957-959

\section{Authors' affiliations}

U Müller-Ladner, Department of Internal Medicine I, University of Regensburg, Germany S Gay, Center for Experimental Rheumatology, Department of Rheumatology, University Hospital Zürich, Switzerland

Correspondence to: Dr U Müller-Ladner Department of Internal Medicine I, University of Regensburg, D-93042 Regensburg, Germany; ulf.mueller-ladner@klinik.uni-r.de

\section{REFERENCES}

1 Tolboom TCA, Pieterman E, van der Laan WH, Toes REM, Huidekoper AL, Nelissen $\mathrm{RGHH}$, et al. Invasive properties of fibroblast-like synoviocytes: correlation with growth characteristics and expression of MMP-1, MMP-3, and MMP-10. Ann Rheum Dis 2002;61:975-80.

2 Wernicke D, Schulze-Westhoff C, Petrow P Bräuer R, Zacher J, Gay S, et al. Stimulation of collagenase- 3 expression in synovial fibroblasts of patients with rheumatoid arthritis by contact with a three-dimensional matrix or with normal cartilage when coimplanted in NOD/SCID mice. Arthritis Rheum 2002;46:64-74

3 Ermis A, Müller B, Hopf T, Hopf C, Remberger $\mathrm{K}$, Jüsten $\mathrm{HP}$, et al. Invasion of human cartilage by cultured multicellular spheroids of rheumatoid synovial cells - a novel in vitro model system for rheumatoid arthritis. J Rheumatol 1998;25:208-13.

4 Müller-Ladner U, Kriegsmann J, Franklin BN, Matsumoto S, Geiler T, Gay RE, et al. 
Synovial fibroblasts of patients with rheumatoid arthritis attach to and invade normal human cartilage when engrafted into SCID mice. Am J Pathol 1996;49:1607-15.

5 Franz JK, Pap T, Müller-Ladner U, Gay RE, Burmester GR, Gay S. T cell independent joint destruction. In: Miossec P, van den Berg WB, Firestein GS, eds. Progress in inflammation research. Vol: $T$ cells in arthritis. Basel: Birkhäuser Verlag, 1998:55-74.

6 Firestein GS, Zvaifler NJ. How important are T cells in chronic rheumatoid synovitis ? II. T cell-independent mechanisms from beginning to end. Arthritis Rheum 2002;46:298-308.

7 Seki T, Selby J, Häupl T, Winchester R. Use of a differential subtraction method to identify genes that characterize the phenotype of cultured rheumatoid arthritis synoviocytes. Arthritis Rheum 1998;41:1356-64.

8 Neumann E, Kullmann F, Judex $M$, Jüsten HP, Wessinghage D, Gay S, et al. Identification of differentially expressed genes in rheumatoid arthritis by a combination of complementary DNA array and RNA arbitrarily primed-polymerase chain reaction. Arthritis Rheum 2002;46:52-63.

9 Firestein GS, Pisetzky DS. DNA microarrays: boundless technology or bound by technology? Guidelines for studies using microarray technology. Arthritis Rheum 2002;46:859-61.

10 Poole AR. Cartilage in health and disease. In Koopman WJ, ed. Arthritis and allied conditions. A textbook of rheumatology. 14th ed. Baltimore: Williams and Wilkins, 2001:226-84.

11 Tomita T, Nakase T, Kaneko M, Shi K, Takahi K, Ochi T, et al. Expression of extracellular matrix metalloproteinase inducer and enhancement of the production of matrix metalloproteinases in rheumatoid arthritis. Arthritis Rheum 2002;46:373-8.

12 Konttinen YT, Ceponis A, Takagi M, Ainola $M$, Sorsa T, Sutinen $M$, et al. New collagenolytic enzymes/cascade identified at the pannus-hard tissue junction in rheumatoid arthritis: destruction from above. Matrix Biol 1998; 17:585-601

13 Pap T, Shigeyama Y, Kuchen S, Fernihough JK, Simmen B, Gay RE, et al. Differential expression pattern of membrane-type matrix metalloproteinases in rheumatoid arthritis. Arthritis Rheum 2000;43:1226-32.

14 Hanemaaijer R, Sorsa T, Konttinen YT, Ding $Y$, Sutinen $M$, Visser $H$, et al. Matrix metalloproteinase-8 is expressed in rheumatoid synovial fibroblasts and endothelial cells. Regulation by tumor necrosis factor- $\alpha$ and doxycycline. J Biol Chem 1997;272:31504-9.

15 Schulze-Westhoff C, Freudiger D, Petrow $P$, Seyfert C, Zacher J, Kriegsmann J, et al. Characterization of collagenase 3 (matrix metalloproteinase 13) messenger RNA expression in the synovial membrane and synovial fibroblasts of patients with rheumatoid arthritis. Arthritis Rheum 1999;42:1517-27.

16 Konttinen YT, Salo T, Hanemaaijer R, Valleala $H$, Sorsa $T$, Sutinen $M$, et al. Collagenase-3 (MMP-13) and its activators in rheumatoid arthritis: localization in the pannus-hard tissue junction and inhibition by alendronate. Matrix Biol 1999;18:401-12.

17 Borghaei RC, Sullivan C, Mochan E. Identification of a cytokine-induced repressor of interleukin-1 stimulated expression of stromelysin 1 (MMP-3). J Biol Chem 1999;274:2126-31

18 Vincenti MP, Coon Cl, Brinckerhoff CE. $\mathrm{NF \kappa B} / \mathrm{p} 50$ activates an element in the distal matrix metalloproteinase 1 promoter in interleukin- $1 \beta$ stimulated synovial fibroblasts. Arthritis Rheum 1998;41:1987-94.

19 Neidhart M, Gay RE, Gay S. Anti-interleukin-1 and anti-CD44 interventions producing significant inhibition of cartilage destruction in an in vitro model of cartilage invasion by rheumatoid arthritis synovial fibroblasts. Arthritis Rheum 2000;43:1719-28

20 Cunnane G, FitzGerald O, Hummel KM, Gay RE, Gay S, Bresnihan B. Collagenase, cathepsin B and cathepsin L gene expression in the synovial membrane of patients with early inflammatory arthritis. Rheumatology (Oxford) 1999;38:34-42.

21 Maeda S, Sawai T, Uziki M, Takahashi Y Omoto $H$, Seki $M$, et al. Determination of interstitial collagenase (MMP-1) in patients with rheumatoid arthritis. Ann Rheum Dis 1995;54:970-5.
22 Ahrens D, Koch AE, Pope RM, Stein-Picarella $M$, Niedbala M. Expression of matrix metalloproteinase 9 (96-kd gelatinase B) in human rheumatoid arthritis. Arthritis Rheum 1996;39:1576-87.

23 Gruber BL, Sorbi D, French DL, Marchese M, Nuovo GJ, Kew RR, et al. Markedly elevated serum MMP-9 (gelatinase B) levels in rheumatoid arthritis: a potentially useful laboratory marker. Clin Immunol Immunpathol 1996:78:161-71.

24 Hummel KM, Petrow PK, Franz JK, Müller-Ladner U, Aicher WK, Gay RE, et al. Cysteine proteinase cathepsin K mRNA is expressed in synovium of patients with rheumatoid arthritis and is detected at sites of synovial bone destruction. J Rheumatol 1998;25: 1887-94

25 Hou W-S, Li W, Keyszer G, Weber E, Levy R, Klein M, et al. Comparison of cathepsins $K$ and $S$ expression within the rheumatoid and osteoarthritic synovium. Arthritis Rheum 2002;46:663-74

26 Pap T, Pap G, Sainsbury I, Franz JK, Hummel $\mathrm{KM}$, Gay RE, et al. In-situ expression of membrane type-matrix metalloproteinase 1 (MT1-MMP) in fibroblasts and osteoclasts at the bone-implant interface of aseptically loosened joint arthroplasties [abstract]. Arthritis Rheum 1998;41(suppl):S362

27 Lewis EJ, Bishop J, Bottomley KMK, Bradshaw D, Brewster M, Broadhurts $M$, ef al. Ro 32-3555, an orally active collagenase inhibitor, prevents cartilage breakdown in vitro and in vivo. $\mathrm{Br} J$ Pharmacol 1997; 121:540-6.

28 Schedel J, Seemayer CA, Zacharias W, Pap $T$, Neidhart M, Kuchen $S$, et al. Targeting cathepsin L by specific ribozymes decreases protein synthesis and cartilage destruction in rheumatoid arthritis [abstract]. Arthritis Rheum 2001;45(suppl):S295.

29 Brown PD. Ongoing trials with matrix metalloproteinase inhibitors. Expert Opin Investig Drugs 2000:9:2167-77.

30 Mudgett JS, Hutchinson NI, Chartrain NA Forsyth AJ, McDonnell J, Singer II, et al. Susceptibility of stromelysin 1-deficient mice to collagen-induced arthritis and cartilage destruction. Arthritis Rheum 1998:41:110-21. 